\title{
Capítulo 2. Sistemas y Pensamiento sistémico
}

\section{Concepto de sistema}

El camino para llegar a los conceptos "sistema", "pensamiento" o "enfoque sistémico" ha estado lleno de dificultades y aún en la actualidad, en muchos campos, no se utiliza con claridad. A pesar de que la concepción sistémica se ha extendido ideológicamente en los distintos campos, la mayoría de estas ideas está basada en popularizaciones y formulaciones filosóficas.

El enfoque sistémico está fundamentado en el concepto de sistema, dicho concepto se puede definir, en su forma más general, de dos maneras: primero, "La teoría de sistemas revoluciono los enfoques de gestión y administración. Estudió las organizaciones como sistemas sociales inmersos en otros sistemas que se interrelacionan y se afectan entre sí" (Hernandez, 2011). Segundo, se entiende que un objeto complejo es un sistema cuando dos o más de sus componentes están interrelacionados e integrados (Bunge, 1995). Los objetos pueden ser individuales o colecciones. La división más radical de las clases de objetos es en objetos o sistemas materiales (naturales o artificiales hechos por el hombre) y objetos o sistemas conceptuales (constructos). Se entiende por "objeto complejo" aquel que contenga dos o más componentes y, en consecuencia, una totalidad es un objeto complejo que puede ser una colección, un agregado, algo indiferenciado o un sistema.

El concepto de relación utilizado en la definición anterior incluye dos tipos distintos que se denominarán "adherencia” (ligadura, vínculo, enlace, conexión o acople). La relación 
de adherencia entre dos objetos materiales implica que por lo menos una de ellas actúa sobre la otra. Las relaciones de adherencia no pueden existir entre elementos conceptuales, como los componentes de un conjunto, porque estos no interactúan. En matemáticas se pueden definir "operaciones" sobre los elementos de un conjunto cuya existencia no los afecta. Pero los componentes de un sistema material no están dados a priori, se construyen (diseñan) o se seleccionan (observación empírica o por hipótesis) durante un proceso de división. Cada sistema ofrece la posibilidad de diversas divisiones, cada división constituye un conjunto, aunque el sistema mismo no es uno de ellos.

El concepto de adherencia permite clarificar la diferencia entre dos totalidades materiales: agregados y sistemas. En efecto, un agregado es una colección de objetos que no están unidos por adherencias y por tanto, le falta integridad o unidad. Es lo opuesto de un sistema. Un objeto es un sistema material, si y solo si, está compuesto de por lo menos dos cosas diferentes conectadas mutuamente, formando una integridad. (Checkland, 1993)

Pese a la división entre clases de objetos, solo se reconocen dos tipos de sistemas: conceptuales y materiales; según sean sus componentes, respectivamente. Conceptuales (preconceptos, proposiciones, teorías, códigos legales, reglas) o materiales por ejemplo (átomos, moléculas, cuerpos, células, organismos, sistema nervioso central, sociedades, empresa de negocios, especies, ecosistema, artefactos). Un sistema funcional conceptual es un conjunto de propiedades (o sus respectivos atributos) relacionadas por leyes o respectivos enunciados legales.

Aunque todo sistema constituye una totalidad, no toda totalidad es un sistema. Solo cuando una totalidad, en algunos aspectos, se comporta como "unidad o totalidad integra", se dice que es un sistema. La integridad de un sistema material se da en grados de adherencia: depende de las conexiones o vínculos entre los componentes del sistema en relación con las acciones desintegradoras del ambiente o por sus contradicciones internas. Un sistema material íntegro es aquel cuyas conexiones entre los componentes son más fuertes que las acciones externas de su ambiente.

Así, se puede decir que "un sistema material es una totalidad íntegra”, que se caracteriza por dos rasgos principales: 1) por la composición, cantidad y calidad de sus componentes integrantes; 2) por las conexiones internas y externas, constantes y estables entre ellos, en virtud de lo cual se unen en un todo único o totalidad íntegra. Este criterio permite diferenciar 
un sistema objetivamente existente de uno que no lo es. Si se tiene el primero y no el segundo, entonces el objeto material es un agregado.

El primer y principal signo del sistema íntegro es que posee cualidades sistémicas integrantes, distintas de las propiedades y cualidades de los componentes que lo integran. En otras palabras, todo sistema material tiene propiedades emergentes no poseídas por sus componentes (y posee por lo menos una de estas propiedades).

Esta propiedad objetiva de los sistemas se resume en la tesis del "emergentismo" ontológico: "Todo sistema posee propiedades distintas a las de sus componentes. Por ejemplo, un hombre tiene propiedades biológicas y propiedades sociales emergentes en el nivel social que no existían en el biológico, tal como su nacionalidad, clase y profesión”. (Herrera, 2007)

Es importante anotar que la ciencia y la tecnología contemporáneas son testimonio de que el mundo que nos rodea no lo forman objetos materiales separados, aislados unos de otros, sino que constituyen un tipo determinado de formaciones sistémicas y de totalidades íntegras. De esta manera, se puede afirmar que los sistemas como totalidades íntegras son realidad objetiva, existen en el mundo, se encuentran en la naturaleza, en la sociedad y, además, son inventados y construidos por el hombre conceptual y materialmente, por medio de las prácticas científicas y tecnológicas (Herrera, 2007).

Es necesario puntualizar que aunque todos los sistemas materiales difieren en características, el problema científico consiste en entender cómo surgen y mantienen su coherencia e integridad, es decir, cuáles son las leyes de su movimiento o cambio. La concepción sistémica no es empirista, pues busca el conocimiento de la totalidad de las relaciones y de las conexiones particulares y universales.

\section{Enfoque sistémico}

La existencia de sistemas y la estructura sistémica de la realidad material, y las formas de su conocimiento, constituyen una esfera del conocimiento que refleja uno de los principales rasgos de la realidad: la capacidad de los procesos y fenómenos de entablar interacciones de tal naturaleza que, como resultado de las mismas, se forman sistemas que otorgan cualidades a los objetos que no tenían inicialmente. De ahí que con el concepto de sistematicidad o de lo sistémico se explica "la propiedad de los objetos de ser o pertenecer a un sistema" (Bunge, 2003). Pues resulta ser una propiedad concreta de los procesos existentes. 
La sistematicidad es la calidad, la propiedad del mundo objetivo que consiste en que el mundo no lo constituyen objetos aislados o agregados, sino conjuntos en interconexión, interacción e integridad. Las cosas dependen y se unen, necesariamente, las unas con las otras (Bunge, 1995). Al respecto, Herrera complementa:

El sistemismo es aquella cosmovisión o enfoque general que considera que todo lo que hay es sistémico y de acuerdo con lo cual el "mundo es un sistema de sistemas" y no un bloque sólido o un agregado de individuos. Esto implica la hipótesis de la existencia de "un sistema estructurado del mundo", se considera el cosmos como el supersistema de todas las cosas cambiables legalmente y nuestro conocimiento de ellas como un supersistema de ideas. (2007)

Considerar la realidad objetiva y su reflejo en el pensamiento desde las posiciones sistémicas, su totalidad y la interacción de las partes que la forman crea un prisma gnoseológico especial, una dimensión que podemos llamar "enfoque sistémico" (o pensamiento sistémico). Esto permite tratar la variedad de los sistemas con propiedades distintas con un marco teórico unificado de sistemas.

El enfoque sistémico y el principio de la sistematicidad, tienen un significado científicoconcreto y filosófico-conceptual, que es un resultado histórico de las ciencias naturales y sociales y cuya comprensión se inició en el marco de la filosofía de la época moderna, en especial con Schelling y Hegel. (Herrera, 2007)

La necesidad del enfoque sistémico para resolver los problemas teóricos y prácticos es producto del propio desarrollo social y de su alta interrelación contemporánea. No es sorprendente que debido al aumento de la complejidad de los problemas que enfrentan las prácticas científicas y tecnológicas, se apliquen cada vez más por diseñadores e investigadores las concepciones sistémicas y se haya incrementado su importancia metodológica.

El pensamiento sistémico como posición filosófica tiene como referentes dos axiomas: el ontológico, que considera que los componentes de la realidad objetiva son sistemas materiales, y el epistemológico, que considera que el conocimiento, como reflejo del pensamiento humano hacia la realidad, está además constituido por sistemas conceptuales. Es decir, el conocimiento debe ser sistémico.

Estos axiomas implican que para explicar los objetos con referencia a la ciencia y la tecnología (para analizarlos, diseñarlos o inventarlos) es necesario considerar sus relaciones o 
su estructura. El enfoque sistémico-estructural ha penetrado en diferentes ramas de las ciencias naturales, siendo las totalidades áreas de estudio, (desde la geografía, la física, la matemática a la sociología), éstas han sido estudiadas usando el lenguaje y las ideas de ésta teoría de sistemas.

\section{El movimiento de los sistemas (MS)}

El programa del movimiento de los sistemas se puede describir como la prueba de que las ideas vistas anteriormente son útiles para plantear el problema de la complejidad organizada que, según los primeros pensadores en este campo, con los métodos de la ciencia normal es difícil de lograr. Fueron Bertalanffy (1976) y Boulding (1956) quienes insistieron en que las ideas emergentes en varios campos podrían generalizarse según el pensamiento de sistemas. "El conjunto de intentos en todas las áreas de estudio por explorar las consecuencias del pensamiento sistémico se puede denominar el “"movimiento de los sistemas”. (Checkland, 1993; Herrera, 2007)

Conceptos como "organización”, "totalidad”, “dirección”, “control”, “autorregulación” y "diferenciación" no eran comunes en ciencias como la física. Muchos reconocen a Bertalanffy (1976) como el fundador del movimiento de sistemas, aunque la fundación de la cibernética por Wiener (1953) fue una contribución esencial de esta época.

Esta nueva rama del conocimiento científico, que nació a partir de la ingeniería, dio como resultado el surgimiento de disciplinas del conocimiento como la informática o la investigación de operaciones, que a su vez dieron gran impulso a la investigación sistemática (Herrera, 2007). Principalmente biólogos y matemáticos, a mediados del siglo XX, elaboraron los principios del enfoque sistémico aplicado a nivel biológico.

Este movimiento es parte de la revolución tecnológico-científica (RTC) de la época actual. Ha producido una creciente transformación de las prácticas científicas y tecnológicas en elementos del desarrollo social y, además, ha influenciado todas las esferas de las actividades humanas. Los cambios cualitativos y cuantitativos en el conocimiento científico se reflejan en los diversos paradigmas que surgen y en la diferenciación e integración de los distintos campos de la cultura.

La tendencia integradora es necesaria para el estudio de los problemas complejos e integrales y para la consideración de las características polisistémicas de la sociedad actual (globalización política y económica del mundo social), situación que obliga a un enfoque sistémico y por tanto a que las prácticas científicas se propongan explicar tales fenómenos y 
que las tecnológicas creen nuevos medios que permitan asegurar la dirección racional de esos objetos y coadyuven a resolver los problemas prácticos de la humanidad. Esta complejidad, marcada por la variedad de componentes, viola la tradicional frontera entre disciplinas y obliga a un enfoque interdisciplinario.

Se puede decir también que la investigación sistémica es una manifestación de la unidad de las funciones investigadoras y transformadoras de carácter integral e interdisciplinario de las ciencias y las tecnologías.

\section{Teorías generales de sistemas (TGS)}

En el campo científico-filosófico la investigación sistémica se desarrolló con la pretensión de encontrar las analogías entre todos los sistemas, estudiando los referentes de explicación como sistemas poseedores de una serie de propiedades comunes, apuntando, como considera Bunge (1995), a que 1) existan conceptos y principios estructurales que se sostengan para sistemas de diferentes clases, y 2) que hayan estrategias de modelación. En particular, el enfoque del “espacio de los estados” que parece trabajar bien en todos los ámbitos.

Específicamente las TGS forman un campo, producto en su mayor parte de las prácticas científicas y tecnológicas, de gran interés para la filosofía, ya que debido a su generalidad esta esfera del conocimiento se traslapa con la ontología. En efecto, la investigación sistémica y la ontología estudian las propiedades comunes a todos los sistemas, no importa su constitución particular y a ambos les interesa investigar las peculiaridades de las teorías extremadamente generales, que son metodológicamente muy diferentes de las teorías específicas. (Bunge, 1995)

De ahí el interés por las teorías generales de sistemas, en las cuales, a juicio de algunos científicos, se encontraba la clave para la síntesis de las ciencias actuales para su unión interior (y no solo para la exterior o formal). La visión de Bertalanffy (1956) apunta a que podría llegarse, como resultado del trabajo en diferentes campos, a una metateoría de los sistemas de alto nivel que se expresaría matemáticamente. Esto llevó históricamente al intento de crear una teoría general de sistemas, llamada a descubrir las leyes más generales que actúan en los sistemas físicos, químicos, biológicos, sociales y técnicos. (Boulding, 1956) 


\section{Teoría general de los sistemas en educación}

En la actualidad, la teoría general de los sistemas tiene una gran incidencia en los diferentes ámbitos educativos: desde la interconexión que se debe dar en las políticas públicas, hasta lo que sucede al interior del aula de clase. La visión sistémica ayuda a reconocer la naturaleza y la dinámica compleja e interdependiente que se da entre los subsistemas educativos, y supone que en su estudio se tienen en cuenta tanto los aspectos cuantitativos como los cualitativos; además, es holística, ayuda a entender el mundo complejo y dinámico que se da al interior del sistema educativo, ya que hace énfasis en ver y analizar todas las relaciones que se suscitan, así como sus interdependencias.

Este nuevo paradigma tiene su propio campo de conocimiento y, a la vez, se nutre de disciplinas como la psicología, la pedagogía, la sociología y la antropología.

Las interdependencias que existen entre el desarrollo educativo, el desarrollo social y el desarrollo económico de nuestras sociedades nos obligan a replantear la visión clásica de las políticas públicas. La política educativa no alcanza los objetivos que normalmente se propone sin una adecuada articulación con las políticas económicas (productivas y distributivas) y sociales (redistributivas). A su vez, las políticas económicas orientadas al crecimiento y a la inserción exitosa de nuestras sociedades en el mercado internacional, requieren de fuertes inversiones en el campo de la producción y distribución social del conocimiento científico y tecnológico. El desarrollo económico no es posible sin desarrollo educativo y éste no es posible sin generación de riqueza y sin una distribución equitativa de la misma. (Tenti, 1999)

Desde la perspectiva de la gestión educativa es importante precisar que, como toda teoría, el pensamiento sistémico tiene sus limitaciones; sin embargo, de sus contribuciones al desarrollo de la teoría contemporánea de las organizaciones - y dentro de ellas, las educativas - han sido importantes los siguientes aspectos:

- El sistema de gerencia estratégica, sus fundamentos, planeación, evaluación y control.

- El concepto de la institución educativa como sistema abierto, en donde priman las alianzas estratégicas y las interdependencias que se dan entre los diferentes componentes (pedagógico, administrativo, directivo y comunidad)

- El concepto de evaluación en términos de impacto social.

- Se han diseñado modelos de calidad para la gestión escolar que tiene como principio orientador el mejoramiento continuo, promoviendo la idea sistemática de incrementar 
la calidad. Cada uno de los procesos puede ser objeto de este principio que se hace operativo a través del diseño, planificación, implementación, corrección y ajuste al proceso y a la evaluación de las prácticas pedagógicas.

- Diseño e implementación de sistemas de gestión de calidad (SGC), que tienen como propósito la coordinación y la articulación de todos los procesos de gestión sobre la base de la comprensión del enfoque sistémico para revisar las interrelaciones entre las dimensiones de cada una de las áreas, de los procesos y resultados.

En esta perspectiva de la gestión educativa, no solo los directivos gestionan, los docentes también realizan funciones desde el aula y dan pasos importantes para recuperar la especificidad de la escuela. Esto conduce al desafío de institucionalizar los procesos de participación que permitan crear equipos de docentes que sean "agentes de cambió".

Según (Asili, 2013) Se hace necesario definir a la institución educativa como una unidad de cambio que debe elaborar su propio proyecto, conforme con la aparición de nuevas funciones y demandas, y con la convicción de que generar en las escuelas propuestas transformadoras e innovadoras debe ser el eje de los cambios para la organización y la gestión educativa.

Desde esta complejidad de la escuela, nacen nuevas exigencias que deben ser abordadas, por lo cual asegurar la gestión institucional en los colegios certificados con un sistema de gestión de calidad es un aspecto esencial para articular las funciones y tareas entre los miembros de la institución. Desde esta perspectiva sistémica, se abordan las políticas educativas, adecuándolas al contexto y a las particularidades y necesidades de cada comunidad educativa.

\section{El contexto escolar y la teoría general de sistemas}

La escuela, como grupo social a partir de su caracterización, se configura como sistema. Al ser tomada como tal, es posible describirla o caracterizarla. La escuela se puede ver como unidad, pero a su vez en ella se encuentran varios subsistemas, como el de evaluación, el de convivencia y el conformado por cada una de las áreas, entre otros. Cada subsistema como componente de la escuela tiene una intencionalidad y aporta, desde su perspectiva, para el logro del objetivo general del sistema, es decir, formar integralmente a cada uno de los estudiantes. Esta sinergia es muy importante para las instituciones educativas, ya que se va consolidando una cultura 
organizacional enfocada a una mayor rentabilidad y competitividad. Además, es importante identificar la relación o comunicación que existe entre los diferentes sistemas o subsistemas. Los subsistemas que conforman el sistema escolar se comunican entre sí y, como lo dice Luhmann (1996), algunos se ponen al servicio de otros, compenetrándose.

La autorreferenciación se interpreta como la autonomía que genera la escuela para la lectura, análisis y evaluación de sus procesos y, desde allí, para generar estrategias imprescindibles para su regulación interna. Sin embargo, en contraposición al planteamiento de Luhmann (1996), es necesario tomar referentes externos para validar y legitimar los procesos implementados en cada uno de los subsistemas.

Según Sacristán (2009), captar la complejidad del mundo y de los problemas educativos, a través del enfoque de sistemas, es un punto de partida metodológico muy interesante. La educación, tanto en su planificación como en su desarrollo concreto a nivel de aula, es compleja y debe ser tratada como un sistema de partes o elementos interrelacionados. Ahora bien, el uso que se ha hecho de los sistemas en educación no ha sido fundamentalmente el de lograr una mejor comprensión de los fenómenos educativos, sus variables o sus complejas interacciones. Se ha usado dentro del marco eficientista con el fin de mejorar la eficiencia del proceso de enseñanza o de aprendizaje.

\section{Los sistemas de gestión y la teoría general de sistemas}

Los sistemas de gestión de calidad están compuestos por varios subsistemas y cada uno está enfocado en la optimización de los diferentes espacios o tareas desarrolladas en la institución. Cada sistema construye su propia estructura a partir de sus componentes y relaciones. Por ejemplo, en el modelo ISO 9001:2000 se pueden identificar los subsistemas que orientan los siguientes procesos: documentación en los establecimientos educativos, responsabilidad de la dirección, política de calidad, enfoque al cliente, autoridad y comunicación, gestión de los recursos, compras, infraestructura, prestación del servicio y control de los dispositivos de seguimiento y medición.

Dela misma manera, el sistema EFQM cuenta con subsistemas que tienen como fin cualificar los diferentes aspectos para satisfacer todas las necesidades y expectativas de sus clientes. Si se transfiere este hecho a la escuela, se puede decir que el sistema EFQM está compuesto por subsistemas que tienen como propósito mejorar las acciones que se implementan al interior del 
colegio en general y al interior del aula. Estas acciones de mejora deben satisfacer plenamente a la comunidad educativa (estudiantes, padres, profesores, personal administrativo).

La teoría de sistemas, aplicada al estudio y funcionamiento de las instituciones educativas, es un instrumento teórico que permite identificar y solucionar problemas que generalmente nacen de la entropía y de las dificultades de adaptación con el entorno y el contexto. Los análisis sistémicos permiten observar los efectos que tiene el trabajo en un componente sobre los demás componentes. Se preocupa por los procesos y los resultados de larga duración, lo que pone de presente la importancia de las historias institucionales. Esto permite, además, la alta participación de los miembros, ya que se requiere complementariedad de perspectivas en torno a la toma de decisiones.

Adoptar la visión sistémica en organizaciones tan complejas como las educativas, supone recordar que su funcionamiento se rige por los principios, ya mencionados, de "totalidad", "globalidad", "correlatividad", "interacción”, "equilibrio dinámico", "entropía”, "sinergia”, “equifinalidad" y "neguentropía”. Cada sistema de gestión de calidad tiene su propia estructura y sus propios componentes de acuerdo a la intencionalidad y especificidad del mismo. Luhmann introduce los aportes de la autopoiesis, elaborada por Maturana y Valera. "Un sistema es autopoiético, en tanto es un sistema que puede crear su propia estructura y los elementos de que se componen" (1996).

Todos los sistemas de gestión de calidad -EFQM, ISO 9001, Malcom Baldrige, Deming, FUNDIBEQ y Galardón a la excelencia- están permeados por conceptos de calidad total. Este referente invita a los directivos del MEN, SED, docentes y padres de familia a realizar un exhaustivo análisis de las nuevas dinámicas que se generan al interior de los colegios para alcanzar exigencias del mercado como: mayor competitividad, productividad, costes, calidad del producto, satisfacción del cliente, entre otros.

Para próximas investigaciones, sería pertinente analizar por qué los diferentes sistemas de calidad que se implementan en el mundo, manejan una lógica de mercado, esto resolvería varias preguntas: ¿la implementación de estos sistemas de gestión en educación garantiza la calidad al interior de las instituciones educativas?, ¿qué está pasando con la formación disciplinar de los docentes y la capacitación frente a lo pedagógico?, ¿antes de implementar estos sistemas de gestión de calidad en los colegios la educación que se impartía era de mala calidad? 